\title{
Flexibility of the Lumbar Intervertebral Disc in Bending and Torsion: Experimental and Finite Element Study
}

\author{
Ramiro Arturo González Gutiérrez, Vladimir Rangel Alonso and Christian Miguel Barraza Armenta \\ Departamento de Metal Mecánica, Instituto Tecnológico de Tijuana, Calzada Tecnológico S/N, Tijuana, B.C. 22414, México
}

\begin{abstract}
Intervertebral disc flexibility is influenced by lifestyle, loading history, trauma, preexisting conditions, age and degeneration. With regard to degeneration, intervertebral discs become less flexible and stiffer. In this study, a testing protocol using bending and torsion loading was developed to gain the flexibility curves and stiffness of ten cadaveric lumbar discs. Measurements of rotation in the sagittal plane (flexion-extension), coronal plane (right-left lateral bending) and transverse plane (torsion) due to a 5 $\mathrm{N}$-m load are reported. Results show that overall normal discs are more flexible and behave in a nonlinear fashion. The testing results were used in a develop finite element model of an intervertebral disc to investigate the stresses and strains in the disc components: annulus fibrosus and nucleus pulposus with regard to degeneration. Simulation of bending and torsion loadings show large strains in the annulus and nucleus from a normal disc, in contrast higher stresses develop in the annulus from a degenerated disc. The proposed methodology is novel, versatile, functional and economic with implications in bioengineering, medical sciences and the clinical field.
\end{abstract}

Key words: Intervertebral disc, flexibility, testing, bending and torsion, finite element method.

\section{Introduction}

Intervertebral disc loading in life involves a combination of tension, compression, torsion and bending. Such loads appear in physiological movements: bending forward, backwards or twisting alone or in combination, such as in running, swimming or lifting [1]. Disc loading in bending, torsion and compression has been investigated, and reported values for radial bulging, axial and rotational stiffness, and flexibility have been gained [2-4]. However, it is not clear how combine loading affects the inside of the disc [5]. Most studies are limited to single loads and only a handful address combine loading with finite element analysis to investigate load sharing and state of stress and strain inside the disc [6, 7]. Since continuous mechanical loading leads to progressive disc deformation, abnormal disc function, and multiple degeneration scenarios [8, 9] intervertebral disc biomechanics becomes relevant to investigate [1, 4]. Thus, the objectives of this study were (1) to evaluate

Corresponding author: Ramiro Arturo González Gutiérrez, Ph.D., research fields: mechanical design, biomechanics, finite element. Email: ramiro.gonzalez@tectijuana.edu.mx. range of motion or flexibility of human cadaveric degenerated discs using a developed loading protocol in bending and torsion, and (2) to investigate the stress and strain distributions inside the disc, particularly the nucleus pulposus and annulus fibrosus using one magnetic resonance imaging based FEM (finite element model) of intervertebral disc degeneration developed by Ref. [10]. The paper is organized as follows: Sections 2-4 give materials and methodologies, Sections 5-6 cover results of testing and simulation, Section 7 addresses the implications and Section 8 gives the main conclusions.

\section{The Specimens}

Five lumbar spines from elderly donors were obtained from Hospital Clinic of Barcelona, Spain. Each spine was further dissected into two motion segments corresponding to lumbar levels L23 and L45. All ten motion segments were inspected to verify the absence of infections, osteoporosis and spondylitis, after which an MRI (magnetic resonance imaging) was done to all segments in accordance with Ref. [11], after which all specimens were stored at $-50^{\circ} \mathrm{C}$. 


\section{Combine Loading Protocol}

Prior to testing, each motion segment was thaw and the posterior elements were removed leaving a vertebra-disc-vertebra segment which was embedded with PMMA into a fixture plate. Testing was done in a MTS Bionix 858 and setup for bending and torsion loading is shown in Fig. 1.

The testing protocol was applied to each disc in accordance with the literature [4, 12, 13] which included a creep period of 15 minutes, bending and torsion moments of $5 \mathrm{~N}-\mathrm{m}$, see Fig. 2.

Bending and torsion angles were measured and disc flexibility and stiffness were calculated. For bending mode, it follows:

$$
\begin{aligned}
& M=0.1 x F \\
& \theta=\sin ^{-1}\left(\frac{Y}{0.1}\right) \\
& K_{B}=\frac{M}{\theta}
\end{aligned}
$$

where, $M$ is the bending moment in $\mathrm{N}-\mathrm{m}, F$ is the applied force of the universal tester machine in $N$ with a lever arm of $0.1 \mathrm{~m}$ (100 mm), $\theta$ is the bending angle in radians, $Y$ is the vertical displacement of the tester, and $K_{B}$ is the bending stiffness of the disc in $\mathrm{Nm} / \mathrm{rad}$ which is applicable in flexion (forward), extension (backward) and right-left lateral bending.

For torsional mode, it follows:

$$
\begin{gathered}
\emptyset=0.0312 Y \\
K_{T}=\frac{T}{\varnothing}
\end{gathered}
$$

where, $\varnothing$ is the torsion angle in radians, $T$ is the torsion moment measure and $K_{T}$ is the torsional stiffness of the disc in Nm/rad.

\section{MRI Base FEM (Finite Element Model) of Disc Degeneration}

A previous develop FEM of disc degeneration for studying disc loading in compression [10] is employed in the present study to investigate stresses and strains in the annulus fibrosus and nucleus pulposus under loadings in bending and torsion. Segmentation of trabecula and cortical bone, annulus, and nucleus from the model is shown in Fig. 3 and a summary of the FEM of disc degeneration is given in Table 1.

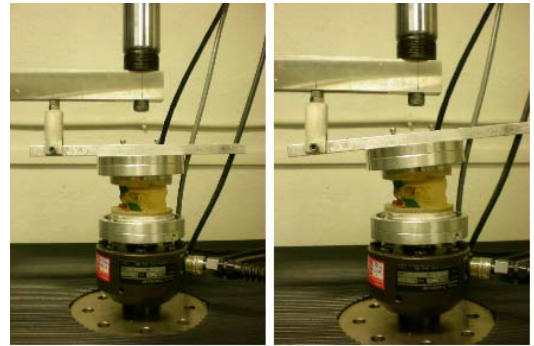

Bending

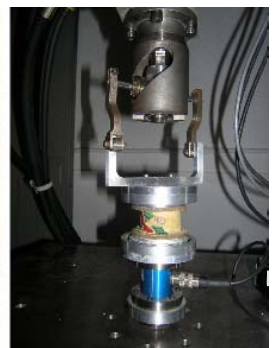

Torsion
Fig. 1 Disc setup for bending and torsion testing.

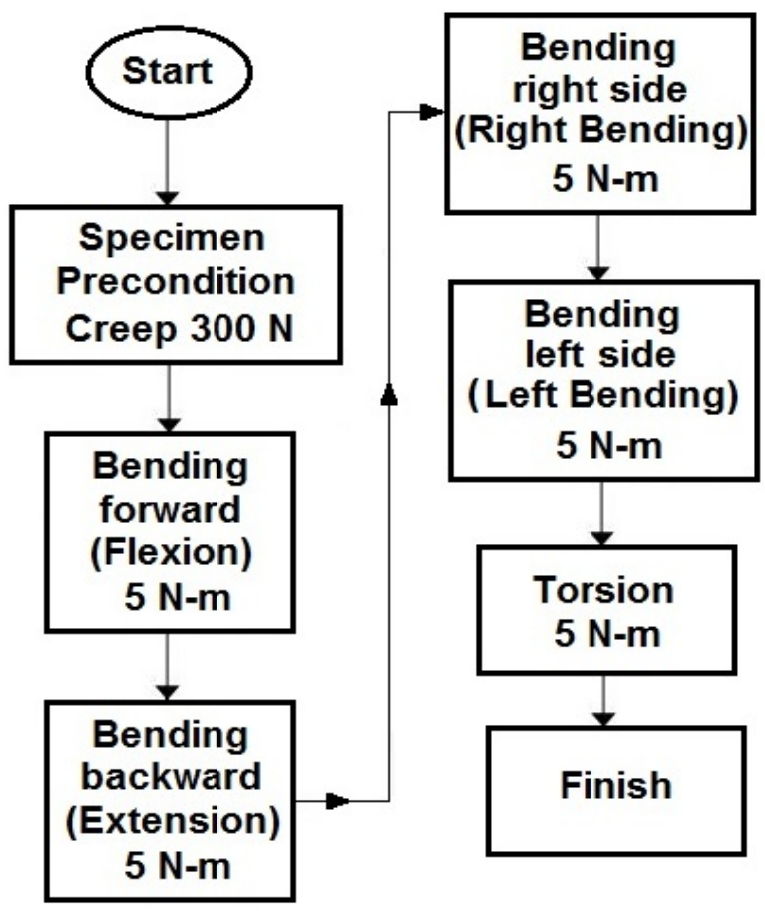

Fig. 2 Combine loading protocol.
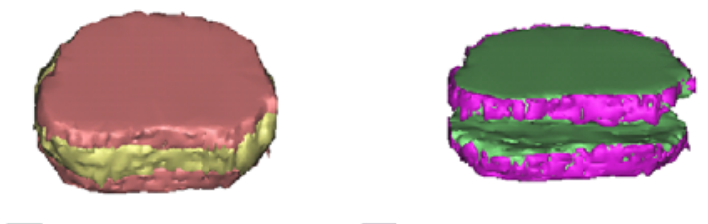

Bone $\square$ Disc

Trabecula Cortial

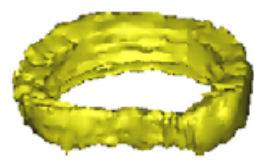

Annulus

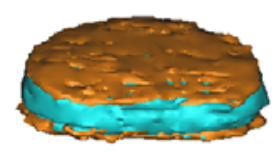

Nucleus

Endplate

Fig. 3 FEM of disc degeneration. 
Table 1 Specifications of the disc model [10].

\begin{tabular}{lll}
\hline Region & $\begin{array}{l}\text { Tetrahedral 4 nodes } \\
\text { element type }\end{array}$ & Material formulation \\
\hline Annulus & 48,091 & Elastomer \\
Nucleus & 45,270 & Elastomer \\
Endplate & 5,508 & Isotropic \\
Cavities & 119 & Isotropic \\
Trabecula & 82,092 & Orthotropic \\
Cortical & 6,666 & Orthotropic \\
\hline
\end{tabular}

Disc materials were treated as incompressible and elastomeric with a formulation based on the Mooney-Rivlin solid model:

$$
W=C_{1}\left(I_{1}-3\right)+C_{2}\left(I_{2}-3\right)
$$

where, $I_{1}$ and $I_{2}$ are the first and second invariant of the Cauchy strain tensor, $C_{1}$ and $C_{2}$ are empiric constants of the disc materials. For the annulus $C_{1}=$ 0.10 and $C_{2}=0.025$, for the nucleus $0.03<C_{1}<0.12$ and $0.01<C_{2}<0.03$ in accordance with Refs. [14, 15].

Bending (i.e. flexion, extension, right and left bending) and torsion simulations were done by implementing a lever arm model and attaching it to the disc model upper side, such that vertical loads will bend and horizontal loads will twist the disc, see Fig. 4.

All simulations were submitted to the same boundary conditions. The type of analysis used was a nonlinear static and the stress and strain in the annulus fibrosus and nucleus pulposus were investigated.

\section{Bending and Torsion Testing}

Results from the bending and torsion testing protocol showed that degenerated discs become stiffer with less flexibility than normal discs. A nonlinear relation between moment load and rotation was found for normal discs, against a linear behavior in degenerated discs. Namely, the angle of rotation of discs under a constant moment load of $5 \mathrm{~N}-\mathrm{m}$ in flexion was $2.4^{\circ}$ to $2.9^{\circ}$, in extension was $2.4^{\circ}$ to $3.1^{\circ}$, and in torsion was $6.7^{\circ}$ to $8.5^{\circ}$, see Fig. 5 .

In right and left lateral bending, there was even results of $2^{\circ}$ for each mode, see Fig. 6. Disc stiffness $(\mathrm{Nm} / \mathrm{rad})$ range between 98 to 120 for flexion, 92 to

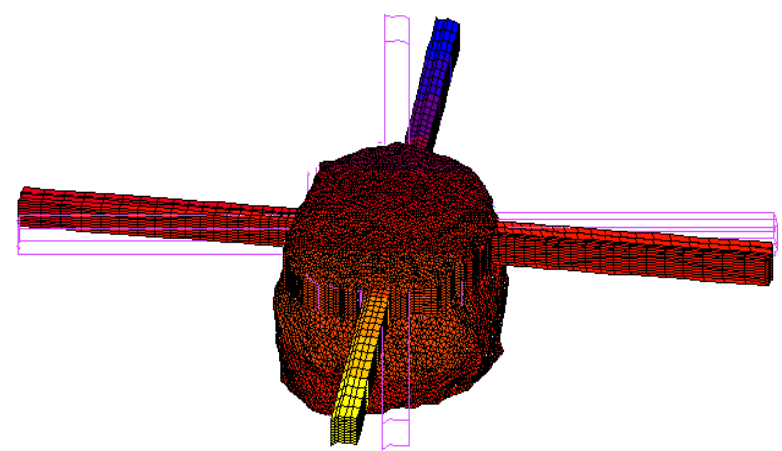

Fig. 4 FEM of disc with lever arms for simulation of bending and torsion loadings.

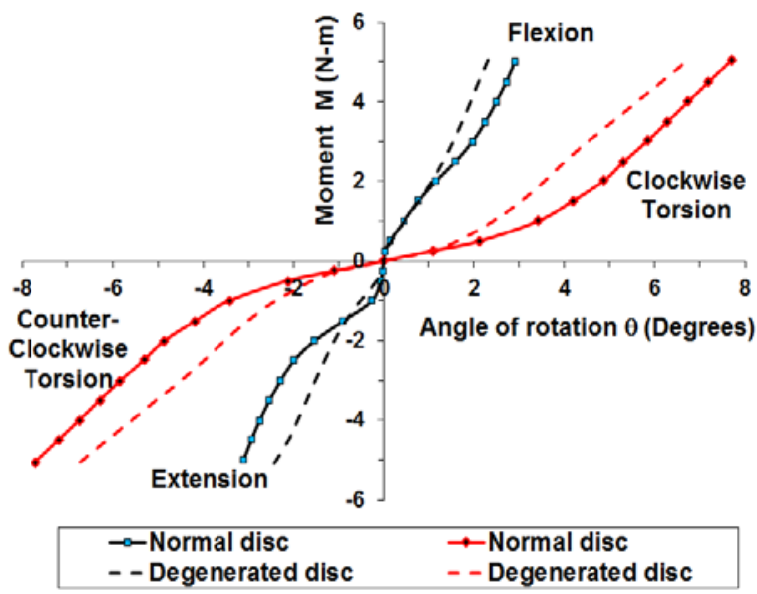

Fig. 5 Mean motion response for disc bending in flexion, extension and torsion $(n=10)$.

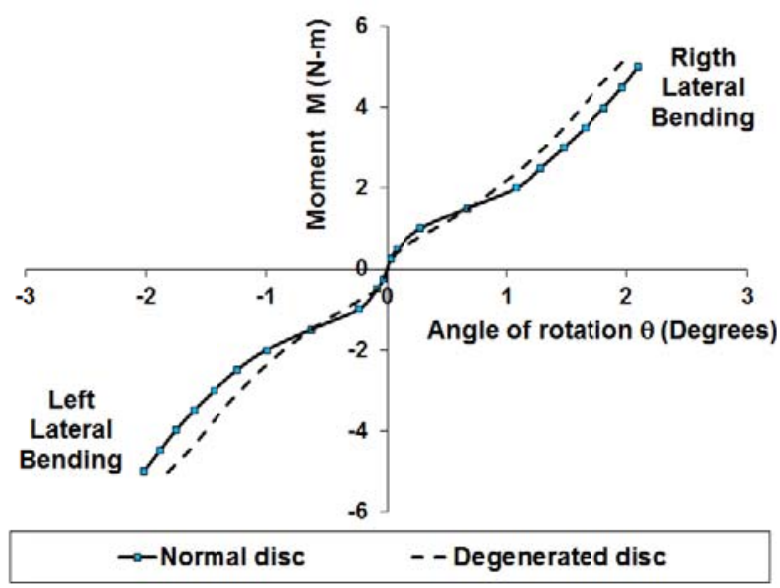

Fig. 6 Mean motion response for disc bending in right and left lateral bending $(n=10)$.

120 for extension, 143 to 161 for right-left lateral bending, and 35 to 42 for torsion. Results show that among degeneration scoring there was no significant differences (student T-test, $p>0.05$ ). 


\section{FE Simulation of Bending and Torsion Loads}

The convergence test for the FEM of disc degeneration gave a convenient element size of 1.5 $\mathrm{mm}$. The Mooney constants selected for the nucleus pulposus were $C_{1}=0.07$ and $C_{2}=0.02$ for a normal disc, and $C_{1}=0.12$ and $C_{2}=0.03$ for a degenerated disc.

In normal discs, bending simulation of $5 \mathrm{~N}-\mathrm{m}$ in flexion, extension, right and left lateral bending causes a principal stress between 200 and $500 \mathrm{kPa}$. These values grew up to $70 \%$ in degenerated discs. The most loaded disc site was the annulus outer layers with principal stress levels reaching $500 \mathrm{kPa}$ and the nucleus with $300 \mathrm{kPa}$. The strain distributions showed

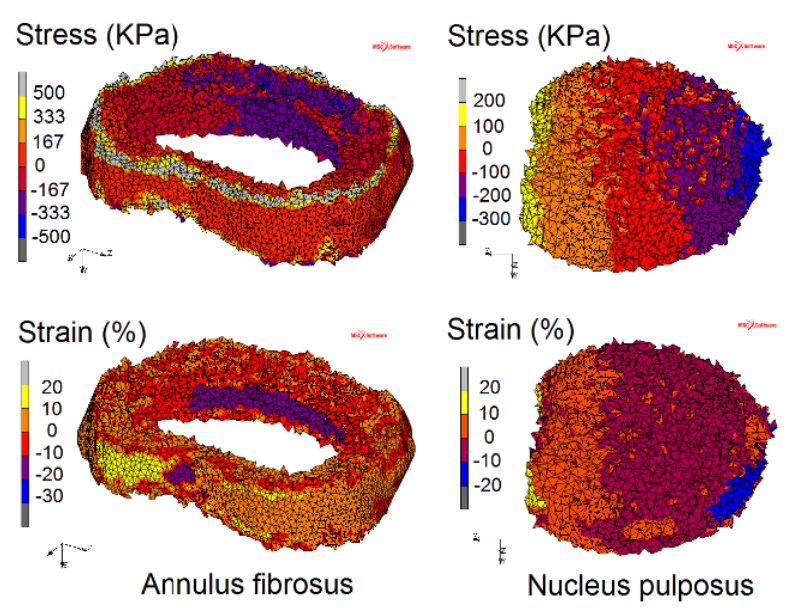

Fig. 7 Stress and strain in the disc annulus and nucleus due to a $5 \mathrm{~N}$-m bending simulation.
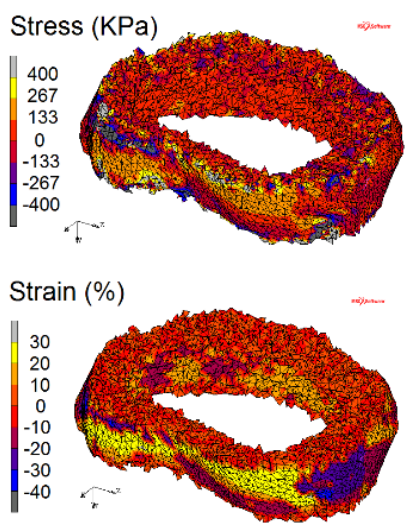

Annulus fibrosus

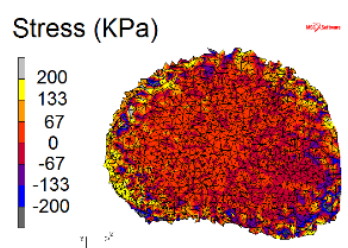

Strain $(\%)$

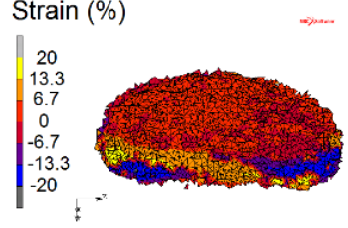

Nucleus pulposus
Fig. 8 Stress and strain in the disc annulus and nucleus due to a $5 \mathrm{~N}$-m torsion simulation. that normal discs deform 33\% to 50\% more than degenerated discs. In flexion, extension, right and left lateral bending the principal strains range between $15 \%$ and $30 \%$ with the largest strains occurring in the annulus outer layers, see Fig. 7.

In torsion simulation, the estimated stress ranges between 125 and $400 \mathrm{kPa}$, and the strain reached values of $40 \%$. Again, the most deformed site was the peripheral annulus and nucleus, see Fig. 8.

\section{Discussion}

Overall, a battery of tests were developed to measure the flexibility of human cadaveric intervertebral discs. Load-displacement curves for cadaveric intervertebral discs reported here are in accordance with literature [3, 12, 13]. Moreover, results show that degenerated discs were $22 \%$ stiffer in flexion, $30 \%$ stiffer in extension, $13 \%$ stiffer in lateral bending and $20 \%$ stiffer in torsion. The increases in bending and torsion stiffness from degenerated discs have been reported elsewhere as the result of excessive formation of bone spurs or osteophytosis, and disc space collapse or osteochondrosis, which in combination stabilize the disc $[16,17]$. That is, bone spurs along the peripheral disc margins will link the two neighboring vertebrae with the collapse disc space causing decrease motion of the spinal segment in bending forward (flexion), backward (extension) or axial rotation (torsion) [8, 18]. Osteophytosis is related to age while osteochondrosis is due to degeneration [8]. In life, flexibility in forward flexion is greater than backward extension which is limited by the vertebrae posterior elements [4], which were removed in this study, as this can explain the even results. Similarly, removal of posterior elements reduces torsion stiffness [13] which can explain the large flexibility. The loss of a nonlinear relationship of load-displacement in bending and torsion from degenerated discs have been related mainly to these pathologies rather than tearing, herniation or disc protrusions alone. However, the 
presence of these latter pathologies which are classified as mild degenerative and its relation with combine loading in bending and torsion is relevant for disc injuries and failure perspectives [2, 19, 20].

Finite element models of intervertebral disc using medical images have been developed [21, 22], but only few studies have also included a testing protocol for collecting input data. The disc model in this study was adequate to perform simulation in bending and torsion loadings in accordance with the testing protocol. The model estimates the stress and strains inside the disc components: annulus fibrosus and nucleus pulposus with respect to degeneration on the basis of input values of the Mooney constants that account for osteophytosis and osteochondrosis, as with other reports [23]. Thus, degenerated discs develop higher stresses while normal discs develop higher strains. Bending simulation shows that flexion, extension, right and left lateral loading causes convex and concave curvature in the disc, which implies combine tensile and compression stresses and higher strains which occurred in the disc posterior side, as reported elsewhere [24-26]. Torsion simulation showed large shearing in the annulus periphery, suggesting a main load carrier upon removal of posterior elements, as previously suggested by Refs. $[7,14,27]$. These findings are relevant because stress and strain concentration sites are source of disc injury and failure, more often in combine loading, such as bending and torsion.

\section{Conclusions}

A methodology has been implemented for the analysis of physiological movement in the lower spine, such as bending down, uprising, twisting or lifting. The technique can be used to investigate other organs or medical devices with implications in bioengineering, life sciences and the clinical field.

\section{Acknowledgement}

We appreciate M.D. Manel LLusa, M.D. Jaume
Pomes (Hospital Clinic, Barcelona, Spain), Dr. Josep Planell (Open University of Catalonia), Dr. Damien Lacroix (Department of Mechanical Engineering, University of Sheffield), our alma mater Instituto Tecnológico de Tijuana (ITT) and the Tecnológico Nacional de México (TecNM) under project: Diseño Industrial y Manufactura TIJ-LGAC-04-16.

\section{References}

[1] Adams, M. A., and Dolan, P. 2005. "Spine Biomechanics." Journal of Biomechanics 38 (10): 1972-83.

[2] Bishop, A., Van Dieën, J. H., Kingma, I., Veen, A. J. V., Jiya, T. U., Mullender, M. G., Paul, C. P. L., Kleuver, M., and Van Royen, B. J. 2013. "Torsion Biomechanics of the Spine Following Lumbar Laminectomy: A Human Cadaver Study". European Spine Journal 22 (8): 1785-93.

[3] Jaramillo, H. E., Puttlitz, C. M., McGluray, K., and García, J. J. 2016. "Characterization of the L4-L5-S1 Motion Segment Using the Stepwise Reduction Method.” Journal of Biomechanics 49 (7): 1248-54.

[4] Newell, N., Little, J. P., Christou, A., Adams, M. A., Adam, C. J., and Masouros, S. D. 2017. "Biomechanics of the Human Intervertebral Disc: A Review of Testing Techniques and Results". Journal of the Mechanical Behavior of Biomedical Materials 69: 420-34.

[5] Walter, B. A., Korecki, C. L., Purmessur, D., Roughley, P. J., Michalek, A. J., and Iatridis, J. C. 2011. "Complex Loading Affects Intervertebral Disc Mechanics and Biology.” Osteoarthritis and Cartilage 19 (8): 1011-18.

[6] Maquer, G., Laurent, M., Brandejsky, V., Pretterklieber, M. L., and Zysset, P. K. 2014. "Finite Element Based Nonlinear Normalization of Human Lumbar Intervertebral Disc Stiffness to Account for Its Morphology.” Journal of Biomechanical Engineering 136 (6).

[7] Azari, F., Arjmand, N., Shirazi-Adl, A., and Moghaddam, T. R. 2017. "A Combine Passive and Active Musculoskeletal Model Study to Estimate L4-L5 Load Shearing." Journal of Biomechanics, http://dx.doi.org/10.1016/j.jbiomech.2017.04.026.

[8] Adams, M. A., and Roughley, P. J. 2006. "What Is Intervertebral Disc Degeneration, and What Causes It?" Spine 31: 2151-61.

[9] Urban, J. P. G., Smith, S. D., and Fairbank, J. C. T. 2004. "Nutrition of the Intervertebral Disc." Spine 29: 2700-9.

[10] Gonzalez Gutierrez, R. A., Rangel Alonso, V., and Zambrano Rosas, J. G. 2015. "Biomechanics of the Intervertebral Disc in Compression: Experimental and Numerical Study.” Journal of Mechanics Engineering 
and Automation 5 (1): 39-44.

[11] Frobin, W., Brinckmann, P., Kramer, M., and Hartwig, E. 2001. "Height of Lumbar Discs Measured from Radiographs Compared with Degeneration and Height Classified from MR Images.” European Journal of Radiology 11: 263-69.

[12] Holewijn, R. M., Faraj, S. S. A., Kingma, I., Royen, B. J., Kleuver, M., and Veen, A. J. 2017. "Spinal Biomechanical Properties Are Significantly Altered with a Novel Embalming Method.” Journal of Biomechanics 55: 144-6.

[13] Guan, Y., Yoganandan, N., Moore, J., Pintar, F. A., Zhang, J., Maiman, D. J., and Laud, P. 2007. "Moment-Rotation Responses of the Human Lumbosacral Spinal Column.” Journal of Biomechanics 40 (9): 1975-80.

[14] Rohlmann, A., Zander, T., Schmidt, H., Wilke, H. J., and Bergmann, G. 2006. "Analysis of the Influence of Disc Degeneration on the Mechanical Behavior of a Lumbar Motion Segment Using the Finite Element Method.” Journal of Biomechanics 39 (13): 2484-90.

[15] Kurutz, M. 2010. "Chapter 9: Finite Element Modelling of Human Lumbar Spine in Finite Element Analysis.” Edited by David Moratal, ISBN 978-953-307-123-7.

[16] Tanaka, N., An, H. S., Lim, T., Fujiwara, A., Jeon, C., and Haughton, V. 2001. "The Relationship between Disc Degeneration and Flexibility of the Lumbar Spine.” The Spine Journal 1 (1): 47-56.

[17] Haugthon, V. M., Lim, T. H., and An, H. 1999. "Intervertebral Disk Appearance Correlated with Stiffness of Lumbar Spinal Motion Segments.” American Journal of Neuroradiology 20 (6): 1161-5.

[18] Boos, N., Weissbach, S., Rohrbach, H., Weiler, C., Spratt, K., and Nerlich, A. 2002. "Classification of Age-Related Changes in Lumbar Intervertebral Disc.” Spine 27 (23): 2631-44.

[19] Costi, J. J., Stokes, I. A. F., Gardner-Morse, M., Laible, J. P., Scoffone, H. M., and Iatridis, J. C. 2007. "Lateral Bending and Flexion Place Intervertebral Disc Tissue at
Greatest Risk of Injury.” Journal of Biomechanics 40 (S2): S596.

[20] Veres, S. P., Robertson, P. A., and Broom, N. D. 2010. "The Influence of Torsion on Disc Herniation When Combined with Flexion.” European Spine Journal 19 (9): 1468-78.

[21] Li, H., and Wang, Z. 2006. "Intervertebral Disc Biomechanical Analysis Using the Finite Element Modeling Based on Medical Images.” Computerized Medical Imaging and Graphics 30: 363-70.

[22] Lodygowski, T., Kakol, W., and Wierszycki, M. 2005. "Three-Dimensional Nonlinear Finite Element Model of the Human Lumbar Spine Segment." Acta of Bioengineering and Biomechanics 7 (2): 17-28.

[23] Schmidt, H., Kettler, A., Rohlmann, A., Claes, L., and Wilke, H. J. 2007. "The Risk of Disc Prolapses with Complex Loading in Different Degrees of Disc Degeneration-A Finite Element Analysis.” Clinical Biomechanics 22 (9): 988-98.

[24] Naserkhaki, S., Jaremko, J., Adeeb, S., and El-Rich, M. 2016. "On the Load-Sharing along the Ligamentus Lumbosacral Spine in Flexed and Extended Postures: Finite Element Study.” Journal of Biomechanics 49 (6): 974-82.

[25] Hu, H. T., Lin, R. M., Kuo, C. S., Lin, P. C., and Huang, K. Y. 2007. "Biomechanical Analysis of the Lumbosacral Spine under Various Loadings and Gestures-A Three-Dimensional Finite Element Study.” Journal of Biomechanics 40 (S2): S271.

[26] Costi, J. J., Stokes, I. A., Gardner-Morse, M., Laible, J. P., Scoffone, H. M., and Iatridis, J. C., 2007. "Direct Measurement of Intervertebral Disc Maximum Shear Strain in Six Degrees of Freedom: Motions That Place Disc Tissue at Risk of Injury.” Journal of Biomechanics 40 (11): 2457-66.

[27] Putzer, M., Ehrlich, I., Rasmussen, J., Gebbeken, N., and Dendorfer, S. 2016. "Sensitivity of Lumbar Spine Loading to Anatomical Parameters." Journal of Biomechanics 49 (6): 953-8. 